\title{
ARTICLE
}

\section{Observation of vibrational overtones by single-molecule resonant photodissociation}

\author{
Ncamiso B. Khanyile, Gang Shu ${ }^{1} \&$ Kenneth R. Brown ${ }^{1}$
}

\begin{abstract}
Molecular ions can be held in a chain of laser-cooled atomic ions by sympathetic cooling. This system is ideal for performing high-precision molecular spectroscopy with applications in astrochemistry and fundamental physics. Here we show that this same system can be coupled with a broadband laser to discover new molecular transitions. We use three-ion chains of $\mathrm{Ca}^{+}$and $\mathrm{CaH}^{+}$to observe vibrational transitions via resonance-enhanced multiphoton dissociation detected by $\mathrm{Ca}^{+}$fluorescence. On the basis of theoretical calculations, we assign the observed peaks to the transition from the ground vibrational state, $v=0$ to $v=9$ and 10. Our method allows us to track single-molecular events, and it can be extended to work with any molecule by using normal mode frequency shifts to detect the dissociation. This survey spectroscopy serves as a bridge to the precision spectroscopy required for molecular ion control.
\end{abstract}

\footnotetext{
${ }^{1}$ Schools of Chemistry and Biochemistry, Computational Science and Engineering, and Physics, Georgia Institute of Technology, Atlanta, Georgia 303320400, USA. Correspondence and requests for materials should be addressed to K.R.B. (email: ken.brown@chemistry.gatech.edu).
} 
P recision spectroscopy of molecules and molecular ions can yield insight into the fundamental physical constants and astrochemical processes ${ }^{1,2}$. Coulomb crystals composed of laser-cooled atomic ions and molecular ions provide a pristine environment for studying the properties of molecules ${ }^{3}$. The laser-cooled atomic ions serve as both a coolant that reduces the temperature and a sensitive detector that allows for single-molecule measurements. The system has been used to study fundamental reaction dynamics ranging from kinetic isotope effects ${ }^{4}$ to observing the relative reaction rates of molecules whose shape differs by the orientation of a single bond ${ }^{5}$. It is also a natural system for precision measurements of molecular ion transitions. Molecular transitions allow for the precise study of fundamental constants using physics that is inaccessible in atomic systems ${ }^{6-8}$. Spectroscopy with Coulomb crystals has been used to directly measure dipole-forbidden transitions in $\mathrm{N}_{2}^{+}$(ref. 9) and accurately determine the rovibrational spectrum of $\mathrm{HD}^{+}$(ref. 10). Future experiments to test the stability of fundamental constants in time have been proposed $^{6}$ using the two-ion-species techniques already exploited in the most precise atomic ion clocks ${ }^{11,12}$.

Expanding these techniques to a wider array of molecular ion species remains a challenge owing to the lack of experimental data on molecular ions transitions. This requires new methods for obtaining spectral information. The spatial localization of molecular ions in a Coulomb crystal results in the required ion density for spectroscopy with low ion numbers relative to traditional techniques. Systems built for high-precision measurement are often incompatible with the survey spectroscopy required to find unknown transitions. However, this is not the case for Coulomb crystals, where the long ion storage time provides multiple opportunities to probe the molecule and the fluorescence of the laser-cooled atomic ion serves as a fast, low-noise detector. $\mathrm{CaH}^{+}$is a candidate molecule for testing the possible variation in the proton-to-electron mass ratio ${ }^{13}$. It is also expected to be relatively abundant in space due to observation of $\mathrm{CaH}$, but has not yet been directly observed ${ }^{14}$. In both cases, laboratory measurements of rovibrational transitions in $\mathrm{CaH}^{+}$are required for scientific progress.

Resonance-enhanced multiphoton dissociation (REMPD) is a common tool in physical chemistry where conditional on a resonant transition a final photon dissociates the molecule. It can be applied to study the vibrations of molecules from diatomics with a single electron ${ }^{15}$ to peptides ${ }^{16}$. Ion fragments are typically detected by an electron multiplier after mass selection ${ }^{16,17}$. Embedding the molecular ions in laser-cooled atomic ions opens up other approaches of detection based on changes in the Coulomb crystal shape ${ }^{18}$ and modulation of the atomic ion fluorescence by driving the resonant motion of the molecular ions ${ }^{15}$. Resolved sideband spectroscopy on a narrow transition of the atomic ions can also be used to identify the fragments ${ }^{19}$. For the case of $\mathrm{CaH}^{+}$, the measurement is simplified by the trapped fragment $\mathrm{Ca}^{+}$fluorescing brightly. This property was used in an ion recycling approach to measure the reaction kinetics of $\mathrm{Ca}^{+}+\mathrm{HD}$ by direct dissociation ${ }^{20}$.

We present the observation of two vibrational overtones of $\mathrm{CaH}^{+}$by two-photon resonant photodissociation of singlemolecular ions sympathetically cooled by two $\mathrm{Ca}+$ ions. The overtone is excited by a mode-locked laser in order to span the thermally occupied rotational states. A second photon dissociates the molecule and the resulting $\mathrm{Ca}^{+}$is detected by a change in fluorescence. The change in the rate of dissociation as a function of the mode-locked laser wavelength reveals two peaks. We assign these peaks to the overtone transtions from the ground vibrational state $v=0$ to $v=9$ and 10 based on theoretical predictions. Our observation is the first measurement of a rovibrational transition in $\mathrm{CaH}^{+}$and shows that singlemolecular ion experiments can be used to discover new transitions.

\section{Results}

Trapping and sympathetically cooling $\mathrm{CaH}^{+}$. We start with loading three ${ }^{40} \mathrm{Ca}^{+}$ions by isotope selective photoionization and then laser cooling the ions to form a three-ion chain. The ${ }^{40} \mathrm{Ca}^{+}$ions are trapped in a radio-frequency Paul trap $\left(r_{0}=0.6 \mathrm{~mm}\right)$ driven at $\Omega=2 \pi \times 14 \mathrm{MHz}$ to confine the ions radially, whereas static DC voltage applied at the endcaps confines the ions axially. The trap is kept at a base pressure of $\sim 4 \times 10^{-9} \mathrm{~Pa}$. The ions are detected by laser-induced fluorescence at $397 \mathrm{~nm}$ onto a photon multiplier tube and electron multiplying charge coupled device camera. A narrow band-pass filter is used to ensure that only $397 \mathrm{~nm}$ light is detected. The experimental apparatus has previously been employed for sympathetic heating spectroscopy ${ }^{21}$ and the sideband cooling of molecular ions ${ }^{22}$.

$\mathrm{A}^{40} \mathrm{CaH}^{+}$molecule is produced by leaking $\sim 5 \times 10^{-7} \mathrm{~Pa}$ of molecular $\mathrm{H}_{2}$ into the chamber via a leak valve. The ${ }^{40} \mathrm{CaH}^{+}$is produced via reactive collisions in the gas phase between ${ }^{40} \mathrm{Ca}^{+}\left(4 P_{1 / 2}\right)$ and the $\mathrm{H}_{2}$ as ${ }^{40} \mathrm{Ca}^{+}+\mathrm{H}_{2} \rightarrow{ }^{40} \mathrm{CaH}^{+}+\mathrm{H}$. The product molecule quickly relaxes to its electronic ground state $\mathrm{X}^{1} \Sigma^{+}$(Fig. 1) and the motion of the molecule is sympathetically cooled by the two $\mathrm{Ca}^{+}$ions. The occurrence of a reaction is determined when one of the ions goes dark and there is a drop in fluorescence counts. Once a reaction occurs, the leak valve is closed and the experiment is delayed until the base pressure is reached. The identity of the molecule can be determined by resolved sideband spectroscopy ${ }^{19}$, and under these experimental conditions we have only observed the formation of $\mathrm{CaH}^{+}$.

A pinhole before the photon multiplier tube allows partial light collection from all three ions and reduces background due to scattered light. Misalignment from the crystal centre results in three distinct collection efficiencies for each ion position. This allows us to detect the position of the dark ion from the fluorescence as shown in Fig. 2. The ion position shifts are due to collision with background gas. Small ion chains of any length could be used to collect the data, and test experiments were performed loading two to four $\mathrm{Ca}^{+}$ions. Three-ion chains were preferred due to faster molecule formation time relative to two ion chains and improved stability relative to four ion chains.

Population of internal states of $\mathrm{CaH}^{+}$. Although the molecular ion is at a translational temperature of a few millikelvin, the

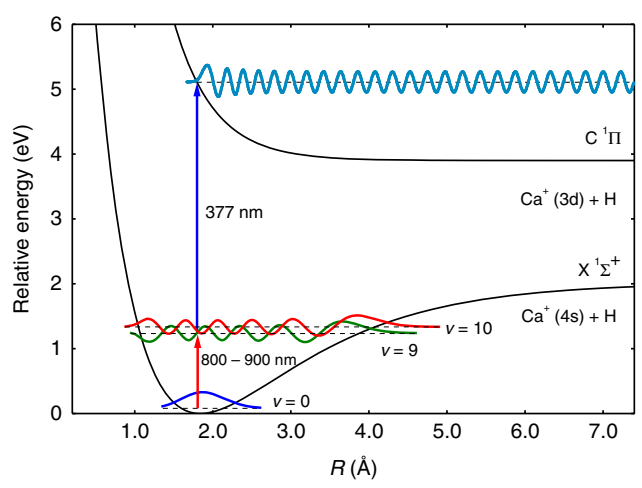

Figure 1 | Energy level diagram of $\mathbf{C a H}^{+}$. Simplified $\mathrm{CaH}^{+}$energy level diagram showing the overtones excited by a pulsed, tunable infrared laser $(800-900 \mathrm{~nm})$. A second ultraviolet laser $(377 \mathrm{~nm})$ excites the overtones to the unbound state to dissociate the molecule. 


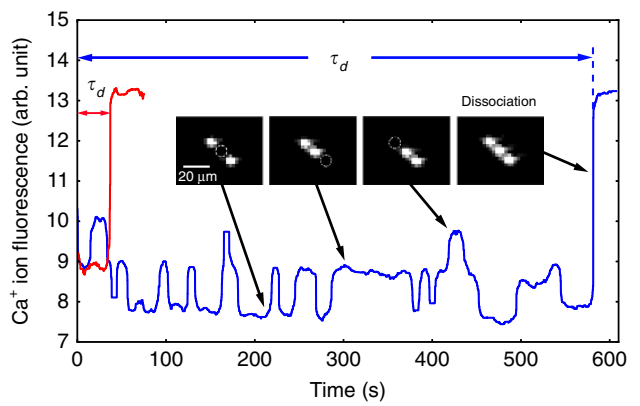

Figure 2 | Dissociation measurement. After reaction with hydrogen, a mixed Coulomb crystal of two $\mathrm{Ca}^{+}$ions and one $\mathrm{CaH}^{+}$ion. The dissociating lasers are applied and the time to dissociation, $\tau_{d}$, is measured by observing the change in fluorescence. The red trace shows an example dissociation event when the infrared laser is resonant with an overtone. The blue trace is an event when the infrared laser is blocked. A pinhole in the optical system allows us to correlate fluorescence with not only the number of $\mathrm{Ca}^{+}$ions but also the relative position of the molecular ion.

internal degrees of freedom are in equilibrium with the room temperature vacuum chamber via black-body radiation. A simplified energy level diagram of $\mathrm{CaH}^{+}$is shown in Fig. 1. The calculated vibrational frequency of the molecule is $1,478.4 \mathrm{~cm}^{-1}$, and we expect that the molecule will be in the ground vibrational state $\mathrm{X}^{1} \Sigma^{+}>99.9 \%$ of the time ${ }^{23}$. On the other hand, the calculated ground state rotational constant, $4.711 \mathrm{~cm}^{-1}$, is small relative to room temperature. The rotational states will be populated with an expected value of $J=5.36$, and the lowest $10 \mathrm{~J}$ states are expected to have $>94 \%$ of the population. Our experiment uses a single molecule at a time, but the black-body radiation will randomize the $J$ state on the order of minutes. To cover this rotational broadening, we use a 150 -fs mode-locked laser to drive the overtones. Alternative approaches include reducing the rotational state space by sympathetic cooling with a buffer gas ${ }^{24}$ or a cloud of laser-cooled neutral atoms ${ }^{25}$ or by preparing the molecular ions in a specific state by threshold ionization ${ }^{18}$. Rotational cooling by optical pumping ${ }^{26-28}$ is currently impossible for $\mathrm{CaH}^{+}$owing to the lack of data on bound transitions.

Single $\mathrm{CaH}^{+}$REMPD. The two-photon photodissociation occurs by first exciting a vibrational overtone of $\mathrm{X}^{1} \Sigma^{+}$and then photodissociating to the state $\mathrm{C}^{1} \Pi$ (Fig. 1). We drive the overtone using of a femtosecond mode-locked spectroscopy laser with $\approx 5 \mathrm{~nm}$ bandwidth, allowing us to ignore the line broadening due to rotational transitions. The second photon comes from a continuous wave laser at $377 \mathrm{~nm}$. The powers of the two lasers are $800 \mathrm{~mW}$ and $200 \mu \mathrm{W}$, respectively. Upon dissociation, the previously dark ${ }^{40} \mathrm{CaH}^{+}$ion will be broken into $\mathrm{Ca}^{+}+\mathrm{H}$, the $\mathrm{Ca}^{+}$will fluoresce again and there will be an increase in fluorescence counts as shown in Fig. 2. For each central wavelength of the spectroscopy laser, we expose the molecular ion to the dissociation lasers and record the time for the molecule to dissociate, $\tau_{d}$. The transition strength is proportional to the rate $r_{d}=1 /\left\langle\tau_{d}\right\rangle$. We repeat the experiment eight times for each wavelength, which resulted in sufficient signal to noise for this survey spectroscopy. Control experiments with the mode-locked laser blocked were used to rule out other processes, including two ultraviolet photon absorption and ultraviolet-induced electron bombardment.

The spectrum clearly shows two peaks that we identify as the $v^{\prime}=10 \leftarrow v=0$ and $v^{\prime}=9 \leftarrow v=0$ overtones of $\mathrm{CaH}^{+}$based on

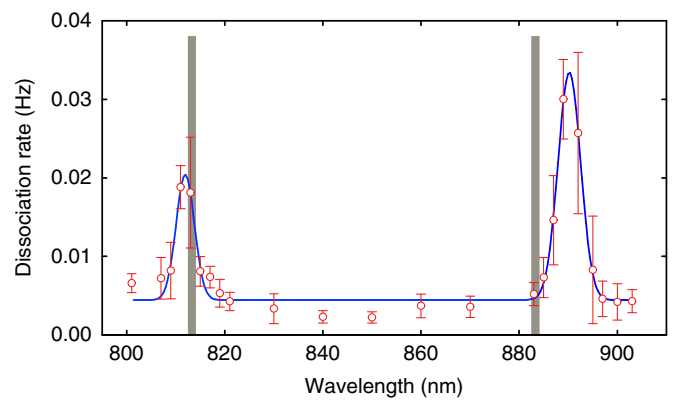

Figure 3 | $\mathbf{C a H}^{+}$vibrational overtone spectra. The measured $\tau_{d}$ are averaged over eight experiments and the inverse is plotted as a function of the IR wavelength. The data reveals two peaks that are fit assuming a Gaussian line shape. Grey bars are centred at the calculated theoretical values $^{23}$ for the $v^{\prime}=10 \leftarrow v=0$ and $v^{\prime}=9 \leftarrow v=0$ overtones. Error bars are the s.e.m. of $r_{d}=1 /\left\langle\tau_{d}\right\rangle, \epsilon_{r}$, propagated from the s.e.m. of $\left\langle\tau_{d}\right\rangle, \epsilon_{\tau}: \epsilon_{r}=r_{d}^{2} \epsilon_{\tau}$.

theoretical calculations using NRel/cc-pCV5Z/CASPT2 (ref. 23; Fig. 3). We measure the $v^{\prime}=10 \leftarrow v=0$ transition to be at $812(3) \mathrm{nm}$ compared with the theoretical value of $813.3 \mathrm{~nm}$ and $v^{\prime}=9 \leftarrow v=0$ transition to be at 890(3) nm compared with the theoretical value of $883.3 \mathrm{~nm}$. This disagreement is in line with observed differences between calculated and measured vibrational transition frequencies in other metal hydrides ${ }^{23}$.

\section{Discussion}

Our experimental setup was intended for high-precision quantum logic spectroscopy ${ }^{11}$ experiments on molecular ions. We have shown that the same setup can be used for the preliminary large range spectroscopy necessary to observe even weak lines despite trapping only a few ions at a time. The next step for precision spectroscopy of $\mathrm{CaH}^{+}$is to reduce the rotational temperature by sympathetic cooling with neutral atoms, and then rotationally resolve these transitions and the fundamental transition ${ }^{24,25}$. Then quantum logic spectroscopy can be performed on ground state cooled $\mathrm{Ca}^{+}{ }_{-} \mathrm{CaH}^{+}$crystals $^{22}$ in order to reach the precision necessary for observing relative changes in fundamental constants ${ }^{12}$.

Our current experiment takes advantage of the fluorescence of the dissociated product and can be easily applied to other fluorescent product ions. The method can be modified for dark product ions by periodically blocking the dissociation laser and measuring the mass of the product ion. This can be achieved by monitoring the sidebands of the atomic ions and calculating the mass of the unknown ion from the observed normal modes ${ }^{19}$. The sideband monitoring can be used to extend few ion crystal spectroscopy beyond REMPD to any action spectroscopy where the laser-cooled atomic ion is inert.

We expect that few ion Coulomb crystals will provide a method for discovering the spectra of ions that are difficult to make in the high numbers required for other spectroscopic methods. As a point of comparison consider the recently reported results on the spectroscopy of $\mathrm{CH}_{5}^{+}$(ref. 29). The spectra requires trapping a few thousand molecular ions per frequency. At equilibrium, $\sim 10$ per cent of those ions form complexes with the He buffer gas, as detected by mass spectrometry. The number of complexes is partially depleted when an internal transition is excited through laser-induced inhibition of complex growth ${ }^{30}$. In the few ion crystal experiment, a single $\mathrm{CH}_{5}^{+}$would be trapped and the normal modes of the crystal monitored. The formation of the $\mathrm{CH}_{5}^{+} \cdot \mathrm{He}$ would result in a change in the normal mode frequencies. The system is in dynamic equilibrium, and for 
every applied laser frequency the normal modes are measured multiple times. The spectral signal is the fraction of measurements where $\mathrm{CH}_{5}^{+} \cdot \mathrm{He}$ is observed. When the excitation laser is on resonance, the signal is suppressed. In principle, one could obtain the entire laser-induced inhibition of complex growth spectra of $\mathrm{CH}_{5}^{+}$using only a single-molecular ion. In practice, the rates of trapping molecules, forming complexes and detecting normal modes will determine when the single-molecule approach is preferable to other methods.

\section{References}

1. Carr, L., DeMille, D., Krems, R. V. \& Ye, J. Cold and ultracold molecules: science, technology and applications. New J. Phys. 11, 055049 (2009).

2. Dulieu, O., Krems, R., Weidemüller, M. \& Willitsch, S. Physics and chemistry of cold molecules. Phys. Chem. Chem. Phys. 13, 18703 (2011).

3. Willitsch, S. Coulomb-crystallised molecular ions in traps: methods, applications, prospects. Int. Rev. Phys. Chem. 31, 175-199 (2012).

4. Staanum, P. F., Højbjerre, K., Wester, R. \& Drewsen, M. Probing Isotope effects in chemical reactions using single ions. Phys. Rev. Lett. 100, 243003 (2008).

5. Chang, Y.-P., Dlugolecki, K., Küpper, J., Rösch, D. \& Willitsch, S. Specific chemical reactivities of spatially separated 3 -aminophenol conformers with cold $\mathrm{Ca}^{+}$Ions. Science 342, 98-101 (2013).

6. Schiller, S. \& Korobov, V. Test of time independence of the electron and nuclear masses with ultracold molecules. Phys. Rev. A 71, 032505 (2005).

7. DeMille, D. et al. Enhanced sensitivity to variation of $\mathrm{m}_{e} / \mathrm{m}_{p}$ in molecular spectra. Phys. Rev. Lett. 4, 043202 (2008).

8. The ACME collaboration, Baron, J. et al. Order of magnitude smaller limit on the electric dipole moment of the electron. Science 349, 269-272 (2014).

9. Germann, M., Tong, X. \& Willitsch, S. Observation of electric-dipoleforbidden infrared transitions in cold molecular ions. Nat. Phys. 10, 820-824 (2014).

10. Bressel, U. et al. Manipulation of individual hyperfine states in cold trapped molecular ions and application to $\mathrm{HD}^{+}$frequency metrology. Phys. Rev. Lett. 108, 183003 (2012).

11. Schmidt, P. O. et al. Spectroscopy using quantum logic. Science 309, 749-752 (2005).

12. Rosenband, T. et al. Frequency ratio of $\mathrm{Al}^{+}$and $\mathrm{Hg}^{+}$single-ion optical clocks; metrology at the 17th decimal place. Science 319, 1808 (2008).

13. Kajita, M. \& Moriwaki, Y. Proposed detection of variation in $\mathrm{m}_{p} / \mathrm{m}_{e}$ using a vibrational transition frequency of a $\mathrm{CaH}^{+}$ion. J. Phys. B At. Mol. Opt. Phys. 42, 154022 (2009).

14. Canuto, S., Castro, M. \& Sinha, K. Theoretical determination of spectroscopic constants of $\mathrm{CaH}^{+}$. Phys. Rev. A 48, 2461 (1993).

15. Roth, B., Koelemeij, J., Daerr, H. \& Schiller, S. Rovibrational spectroscopy of trapped molecular hydrogen ions at millikelvin temperatures. Phys. Rev. A 74, 040501 (2006).

16. Rizzo, T. R., Stearns, J. A. \& Boyarkin, O. V. Spectroscopic studies of cold, gas-phase biomolecular ions. Int. Rev. Phys. Chem. 28, 481-515 (2009).

17. Schneider, C., Schowalter, Chen, K., Sullivan, S. T. \& Hudson, E. R. Lasercooling-assisted mass spectrometry. Phys. Rev. Appl. 2, 034013 (2014).

18. Tong, X., Winney, A. H. \& Willitsch, S. Sympathetic cooling of molecular ions in selected rotational and vibrational states produced by threshold photoionization. Phys. Rev. Lett. 105, 143001 (2010).
19. Goeders, J. E. et al. Identifying single molecular ions by resolved sideband measurements. J. Phys. Chem. A 117, 9725-9731 (2013).

20. Hansen, A. K., Sørensen, M. A., Staanum, P. F. \& Drewsen, M. Single-ion recycling reactions. Angew. Chem. Int. Ed. 51, 7960-7962 (2012).

21. Clark, C. R. et al. Detection of single-ion spectra by Coulomb-crystal heating. Phys. Rev. A 81, 043428 (2010).

22. Rugango, R. et al. Sympathetic cooling of molecular ion motion to the ground state. New J. Phys. 17, 035009 (2015).

23. Abe, M., Kajita, M., Hada, M. \& Moriwaki, Y. Ab initio study on vibrational dipole moments of $\mathrm{XH}^{+}$molecular ions: $\mathrm{X}={ }^{24} \mathrm{Mg},{ }^{40} \mathrm{Ca},{ }^{64} \mathrm{Zn},{ }^{88} \mathrm{Sr},{ }^{114} \mathrm{Cd}$, ${ }^{138} \mathrm{Ba},{ }^{174} \mathrm{Yb}$ and ${ }^{202} \mathrm{Hg}$. J. Phys. B At. Mol. Opt. Phys. 43, 245102 (2010).

24. Hansen, A. K. et al. Efficient rotational cooling of Coulomb-crystallized molecular ions by a helium buffer gas. Nature 508, 76-79 (2014).

25. Rellergert, W. G. et al. Evidence for sympathetic vibrational cooling of translationally cold molecules. Nature 495, 490-494 (2013).

26. Staanum, P. F., Højbjerre, K., Skyt, P. S., Hansen, A. K. \& Drewsen, M. Rotational laser cooling of vibrationally and translationally cold molecular ions. Nat. Phys. 6, 271-274 (2010).

27. Lien, C.-Y. et al. Broadband optical cooling of molecular rotors from room temperature to the ground state. Nat. Commun. 5, 4783 (2014).

28. Schneider, T., Roth, B., Duncker, H., Ernsting, I. \& Schiller, S. All-optical preparation of molecular ions in the rovibrational ground state. Nat. Phys. 6 , 275-278 (2010).

29. Asvany, O. et al. Experimental ground-state differences of $\mathrm{CH}_{5}^{+}$. Science 347, 1346-1348 (2015).

30. Chakrabarty, S. et al. A novel method to measure spectra of cold molecular ions J. Chem. Phys. Lett. 4, 4051-4054 (2013).

\section{Acknowledgements}

We thank M. Abe, S. Janardan, M. Kajita and E. Hudson for their useful discussions, Y. Choi for assistance with data collection and D. Denison for use of the MIRA. This work was supported by ARO grant W911-NF-12-1-0230.

\section{Author contributions}

K.R.B. and N.B.K. conceived the experiment and wrote the manuscript. N.B.K. and G.S. collected the data. K.R.B., N.B.K. and G.S. performed the data analysis and prepared the figures.

\section{Additional information}

Competing financial interests: The authors declare no competing financial interests.

Reprints and permission information is available online at http://npg.nature.com/ reprintsandpermissions/

How to cite this article: Khanyile, N.B. et al. Observation of vibrational overtones by single-molecule resonant photodissociation. Nat. Commun. 6:7825 doi: 10.1038/ncomms8825 (2015).

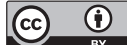

This work is licensed under a Creative Commons Attribution 4.0 International License. The images or other third party material in this article are included in the article's Creative Commons license, unless indicated otherwise in the credit line; if the material is not included under the Creative Commons license, users will need to obtain permission from the license holder to reproduce the material. To view a copy of this license, visit http://creativecommons.org/licenses/by/4.0/ 